\title{
Aptitud de médicos residentes para la búsqueda y recuperación de información
}

\author{
Maria del Rubí Castro-Montoya* \\ Mariano Padilla-Mendoza* \\ José Manuel Ornelas-Aguirre**
}

Artículo recibido:

2 de diciembre de 2013

Artículo aceptado:

28 de octubre de 2016

\section{Resumen}

Se evaluó el efecto de una estrategia educativa para el desarrollo de la aptitud de los médicos residentes en la búsqueda y recuperación de documentos electrónicos. De igual manera se describió el comportamiento informacional al buscar y recuperar documentos electrónicos, anterior y posterior a la alfabetización informacional (ALFIN). El método consistió en un estudio cuasiexperimental de intervención, con una selección de la población a través de censo, conformada por médicos residentes del ciclo 2010-2013 y 2010-2014. Se realizó una medición antes y después de la ALFIN para identificar diferencia significativa de aptitud, antes y después del otorgamiento de la

* Instituto Mexicano del Seguro Social, Sonora, México

maria.castromo@hotmail.com mariano.padilla@imss.gob.mx

** Universidad de Sonora, México_ jose.ornelas@cajeme.uson.mx

INVESTIGACIÓN BIBLIOTECOLÓGICA, vol. 32, núm. 75, abril/junio, 2018, México, ISSN: 2448-8321 pp. 145-161 
estrategia educativa. El análisis general del instrumento anterior y posterior muestra un cambio positivo demostrado por un ascenso en la aptitud de un $20 \%$ a un $35 \%(Z=-3.46$ con una $\mathrm{p}=0.01)$ entre las pruebas anterior y posterior. Se obtuvieron importantes datos para medir el comportamiento informacional de los residentes de medicina en la búsqueda y recuperación de información. En conclusión, los usuarios capacitados adquieren la aptitud necesaria para buscar y recuperar los artículos de revistas y los libros electrónicos a través del IMSS, una vez que se les otorga la ALFIN sobre búsqueda y recuperación de información.

Palabras clave: Comportamiento Informacional; Búsqueda de Información; Aptitud; Alfabetización Informacional

Aptitude for medical residents to the search and recovery of information

Maria del Rubi Castro-Montoya, Mariano Padilla-Mendoza and José Manuel Ornelas-Aguirre

\section{Abstract}

This study examines the effect of an educational strategy for the development of electronic document search and retrieval skills in medical students performing residency requirement. Additionally, the information behaviors of search and retrieval of electronic documents before and after information literacy remediation are described. This quasi-experimental intervention study employs the census of residents enrolled in the 2010-2014 and 2010-2013 cycles. Measurements were performed before and after information literacy remediation in order to identify significant differences in aptitude before and after the execution of said remediation. Results of the pre- and post-measurements show a positive change with aptitude rising from $20 \%$ to $35 \%(\mathrm{Z}=-3.46$ with $\mathrm{p}=0.01$ ). Important data were obtained to measure the informational behavior of medical residents in information retrieval. We conclude that users trained in IL acquire the necessary skills to search and retrieve journal articles and e-books offered by IMSS.

Keywords: Information Behavior; Information Seek; Aptitude; Information Literacy 


\section{INTRODUCCIÓN}

— $\mathrm{n}$ el presente estudio se evalúa el efecto de una estrategia educativa como - $p$ parte de la alfabetización informacional (ALFIN) otorgada en un centro de documentación, con lo cual se busca medir el desarrollo de la aptitud de los médicos residentes para la búsqueda y recuperación de documentos electrónicos. De igual manera se describe el comportamiento informacional al buscar y recuperar documentos electrónicos, anterior y posterior a la ALFIN.

Los estudios de comportamiento informativo donde se pueda analizar la aptitud del usuario al otorgarle una alfabetización informacional son de gran conveniencia para las unidades de información. A través de este tipo de investigaciones se puede conocer si el usuario realmente adquiere las habilidades para la búsqueda y recuperación de información una vez que se le ha brindado la ALFIN.

El Instituto Mexicano del Seguro Social, a través de su sistema bibliotecario (SIBIMSS), busca contar con recursos documentales que apoyen la actualización de su personal de las áreas de la salud, pero además pretende que estos recursos sean oportunos y pertinentes para dicho personal, al grado de apoyarlo en las decisiones que le servirán para brindar una mejor calidad de vida para la población a la que sirve.

\section{Formación de usuarios en las unidades de información}

Los médicos, como profesionales de la salud, tienen probablemente más que cualquier otra profesión el compromiso de renovar sus conocimientos durante toda su vida laboral, puesto que los constantes avances de la medicina les exigen mantenerse actualizados para contribuir a la mejora de sus pacientes y en general a la calidad de vida de la población que atiende el sistema de salud (De la Cruz Solis, González Méndez y Vivás Jiménez, 2007: 3). De allí la importancia de que a estos médicos se les instruya en el mejor mecanismo para acceder a la información, a fin de aprovechar cualquier fuente que les pueda servir para esta actualización.

La constante necesidad de información de los usuarios y la variedad de formatos para conservar y diseminar dicha información han obligado a las bibliotecas y a otras unidades de información (UI) a reconocer la importancia de educar y formar usuarios en el manejo de catálogos, resúmenes y bibliografías en todos los soportes existentes, lo cual ha generado que los profesionales de la información perciban al médico como un usuario que requiere orientación y asesoría oportunas (López Espinosa y Díaz del Campo, 1996). 
El papel educativo de las bibliotecas tiene sus inicios en los años 70 en el ámbito anglosajón. La formación de usuarios tradicional de los años 80 y anteriores sólo enseñaba a usar herramientas de información de tipo librario como libros, revistas, índices, catálogos, etc., sin explorar actividades más complejas como la búsqueda y recuperación de dicha información, el pensamiento crítico y la evaluación de lo que los usuarios consideran. Como señala Licea (2007: 215-232), el bibliotecario como segmento de la alfabetización informacional (Information Literacy) tiene la obligación de evaluar esa alfabetización para tomar decisiones más adecuadas a las necesidades de sus usuarios.

\section{La ALFIN en los programas de formación de usuarios}

El interés por educar y formar usuarios en la búsqueda y recuperación de la información incluye ofrecer un conjunto de aptitudes para localizar, manejar y utilizar la información de forma eficaz y oportuna, a fin de que se pueda ofrecer al usuario del área de la salud la posibilidad de mantenerse actualizado en los estudios relacionados con la especialidad de su interés y así buscar una mejor calidad de vida en beneficio de sus pacientes.

La American Library Association (ALA) ha definido la ALFIN como la perspectiva de aprender a aprender. Ésta incluye la habilidad para localizar, gestionar, evaluar críticamente y utilizar la información para resolver problemas, para la investigación, la toma de decisiones y la formación continua (Universidad Complutense de Madrid y Pérez Iglesias, 2007). En nuestros días, se vuelve necesario que la aportación que hace la biblioteca a este cambio sea de tránsito de la información, donde el usuario sea capaz de conocer las fuentes, los recursos y las herramientas informativas que requiere aun cuando no tenga siempre la ayuda del bibliotecario, puesto que éste ya le brindó la capacitación para ello. Hoy no se trata de apropiarse de cuanto documento existe, sino más bien de las estrategias que empleemos para localizar aquellos documentos que realmente cubren una necesidad de información (Perello, 2005: 48); estrategias que deberán formularse de una manera más eficiente ante los nuevos soportes documentales.

Los Centros de Documentación, como en el que se desarrolla este estudio, son unidades de información (UI) que buscan fortalecer la investigación y la educación continua para que se pueda adquirir la responsabilidad de mantenerse actualizado en los cambios y exigencias que se van suscitando con las tecnologías de la información y la comunicación (TIC). Resulta prioritario crear servicios de información que permitan optimizar la utilización de las herramientas y así conocer qué se investiga, cómo buscarlo y cómo agilizar su obtención. 
Ante este panorama, desde 2006 el Instituto Mexicano del Seguro Social (IMSS) ha logrado desarrollar programas de formación de usuarios en su sistema bibliotecario (SIBIMSS), por lo que se vuelve necesario evaluar la formación que cada Centro de Documentación en Salud (CDS), sala de lectura o sala de consulta electrónica del IMSS están brindando al usuario para adquirir las habilidades que le permitan utilizar de forma oportuna los recursos documentales electrónicos (RDE) a través de la plataforma digital de la Coordinación de Educación del mismo instituto (Lancaster, 2003: 239-274).

Autores como Lancaster (2003), que se encuentran interesados en la evaluación de las bibliotecas y sus diferentes servicios y recursos, señalan la importancia de crear y mantener la evaluación a los programas de formación de usuarios ya que permite medir diversos aspectos como la eficacia o el costo-beneficio, realizando micro o macro evaluaciones dependiendo de los objetivos de la evaluación del programa. Este tipo de evaluaciones puede realizarse también subjetiva u objetivamente a través de cuatro pasos que pueden seguirse en forma individual o conjunta:

1. La reacción de los participantes.

2. El aprendizaje adquirido.

3. Los cambios en el comportamiento de los usuarios.

4. Los resultados del programa.

\section{Comportamiento informacional en la búsqueda de información}

Algunos estudios, como el de Llanusa et al. (2015: 339), expresan la necesidad de evaluar el comportamiento informacional de los usuarios para diagnosticar el uso de la información que buscan los médicos como parte del apoyo a su práctica profesional. La autora expone las dificultades que dichos usuarios encuentran al buscar y recuperar las fuentes que cubren sus necesidades de información. Otros trabajos, como el realizado por Gonzáles Teruel (2011), han estudiado el comportamiento informacional desde sus teorías, métodos o técnicas, y concluyen que este tipo de investigaciones cada día tienen mayor impacto, generándose así un aspecto importante a considerar para realizar estos estudios.

Se han descrito algunas evaluaciones que permiten determinar el grado de conocimiento o la aptitud que el usuario adquiere, realizándole evaluaciones pre y post formación (Licea, 2007: 215-232), inclusive hay estudios que señalan que el usuario realmente adquiere la aptitud de mejoramiento una vez que se le ha otorgado la capacitación para utilizar las Bases de Datos (BD) lo cual permite medir a corto, mediano y largo plazo los beneficios que el 
usuarios adquiere junto a la ALFIN. Como señala Viniegra Velázquez (2002: 132), los bibliotecarios que ofrecen la educación al usuario deben buscar motivarlo en la realización de búsquedas de información, aun cuando en determinado caso no fuese sólo para cubrir algún aspecto académico, sino para el aprovechamiento de un recurso documental electrónico creado para la utilización de los empleados del IMSS, ya que sus objetivos se orientan principalmente a fomentar la salud de la comunidad de México y este objetivo está inmerso en la educación continua de la Coordinación de Educación a la que pertenecen los Centros de Documentación del Seguro Social.

\section{Descripción de la ALFIN que ofrece el CRDS de Sonora}

El curso de formación de usuarios que representa la ALFIN, o estrategia educativa participativa que se ofrece a los usuarios de un Centro de Documentación en Salud en México, se imparte desde 2006. En este curso se difunden los objetivos y características del curso además del perfil del usuario a quien va dirigido. Los contenidos del curso abordan desde enseñar al usuario la dirección electrónica de la plataforma digital que contiene los recursos documentales, conocida como EDUMED (de la abreviatura EDUcación MEDica), así como la identificación de la contraseña y nombre de usuario para poder ingresar a la página electrónica. Posteriormente se van explorando los portales uno por uno, navegando a través de los hipervínculos que se deberían utilizar para realizar una búsqueda dirigida con base en un tema de búsqueda específico y condicionando, cuando es posible, el idioma, el tipo de documento y el rango de tiempo de los documentos que se quieren localizar (Castro Montoya, 2008).

De igual manera se expone que los objetivos de esta estrategia o ALFIN son ofrecer a los usuarios el conocimiento que los vuelva aptos para aprovechar el RDE a través de EDUMED, a fin de motivar la habilidad de buscar y obtener los artículos y libros electrónicos que cubran sus necesidades de información y que están disponibles a través de este portal del IMSS.

\section{Metodología}

El diseño cuasiexperimental fue realizado en el Centro Regional de Documentación en Salud (CRDS) del Hospital Regional No. 1 del IMSS en Ciudad Obregón, Sonora, cuya clave de identificación es SON00R. La población de estudio fueron los médicos residentes que cursaron las diferentes especialidades adscritas al módulo hospitalario conformado por el Hospital 
General Regional No. 1, la Unidad Médica de Alta Especialidad y la Unidad de Medicina Familiar No. 1. Se utilizó un muestreo de tipo no probabilístico a través de censo, donde se incluyeron sólo a los residentes de Ortopedia, Urgencias Médico-Quirúrgicas y Medicina Familiar de acuerdo a los criterios de selección. Los grupos fueron de las generaciones 2010-2013 y 2010-2014. Consideramos relevante mencionar que la ALFIN se impartió en su primer grado de estudios, pues como señala la literatura analizada, se obtienen mejores resultados cuando otorgamos esta capacitación al usuario al inicio de su formación académica.

La ALFIN evaluada fue el curso de capacitación en la búsqueda y recuperación de documentos en la página electrónica del CDRS en Sonora. Esta estrategia educativa consistió en aplicar diferentes técnicas destinadas a optimizar la consulta en la plataforma digital y las BD allí ubicadas, sea en forma interactiva o diferida, para seleccionar y recuperar los registros relevantes a las necesidades de información del usuario (IMSS, 2009). Una vez que se contó con cada grupo a capacitar, se proporcionó antes y después de la ALFIN un cuestionario o instrumento de medición válido y confiable que permitió medir la capacidad de los médicos residentes ante la búsqueda y recuperación de documentos en la página electrónica de la Coordinación de Educación (CDES) del IMSS.

El instrumento de medición constó de 7 indicadores representados con 5 casos problematizados sobre el acceso y uso de una búsqueda de información dentro de la plataforma digital de la CDES. Los casos problematizados fueron creados con base en el procedimiento estándar de atención a los usuarios en el CRDS. El usuario tenía la opción de elegir una de tres respuestas: Falso, Verdadero y No lo sé.

Los indicadores utilizados en la instrumento fueron los siguientes: 1) conocimiento que se tiene de la dirección electrónica http://edumed.imss.gob. $\mathrm{mx} ; 2$ ) ingreso a las bases de datos (BD) que contiene el portal de EDUMED, a través de los pasos para el acceso; 3) conocimiento para realizar una búsqueda básica y avanzada, enfatizando la diferencia que se produce al realizar las búsquedas en las áreas de título y las del resto del documento; 4) capacidad para aprovechar los términos MeSH (Medical Subject Heading) de Medline y la combinación con los operadores booleanos para limitar la búsqueda mediante uniones, disyunciones, truncamientos, etc.; 5) conocimiento para el préstamo de libros digitales, y 6) recuperación de artículos del CRDS en texto completo.

El instrumento fue validado en relación a su contenido por cuatro bibliotecarios expertos, evaluando la claridad de los enunciados que conformaron 
la encuesta. Se eligieron aquellos enunciados aprobados por tres de los cuatro evaluadores. Posteriormente, se solicitó una validación de constructo a un grupo de expertos en metodología de investigación, mismos que analizaron los aspectos que debía contemplar dicho instrumento y entregaron su carta aprobatoria. Por último, se realizó una prueba piloto a un grupo de 10 usuarios y se analizó a través de la prueba de Wilcoxon para dos muestras relacionadas $(\mathrm{Z}=2.706 ; \mathrm{p}=0.007)$.

Toda la información se capturó en una base de datos en el paquete estadístico SPSS versión 15.0 para Windows. Para el análisis estadístico de los resultados se utilizó una escala de medición de tipo ordinal, utilizando al sujeto estudiado como su propio control, con la cual se buscó identificar la existencia de diferencias estadísticamente significativas entre la evaluación anterior y posterior a la ALFIN, analizada mediante la prueba de Wilcoxon para dos muestras relacionadas. Un valor de $p$ igual o menor a 0.05 fue considerado como estadísticamente significativo.

\section{Presentación y anÁlisis de Resultados}

Se aplicó la intervención a 39 estudiantes de posgrado que estudiaron las especialidades de Ortopedia (3), Urgencias Médico-Quirúrgicas (18) y Medicina Familiar (18). Se estudiaron 6 indicadores con 12 variables que permitieron evaluar el efecto de la estrategia educativa aplicada, a fin de buscar un aumento en la aptitud de los médicos residentes para la búsqueda y recuperación de artículos y libros electrónicos a través del portal de EDUMED.

Los resultados de la Tabla 1 manifiestan que al codificar los datos del instrumento anterior y posterior se observa un cambio positivo de aptitud al ascender 69 puntos y se modifica la mediana para el antes y el después $(\mathrm{Z}=-3.46, \mathrm{p}=0.01)$. Esta modificación de aptitud se puede ver mejor reflejada en el diagrama de caja de la Figura 1.

\begin{tabular}{|c|c|c|c|c|c|c|}
\hline $\begin{array}{c}\text { Aplicación del } \\
\text { instrumento }\end{array}$ & $\begin{array}{c}\text { Datos } \\
\text { codificados }\end{array}$ & Mínimo & Q1 & Q2 & Q3 & Máximo \\
\hline Antes & 93 & -6.00 & 1.00 & 2.00 & 4.00 & 10.00 \\
\hline Después & 162 & -1.00 & 2.00 & 4.00 & 6.00 & 12.00 \\
\hline
\end{tabular}

Tabla 1. Codificación de los datos 


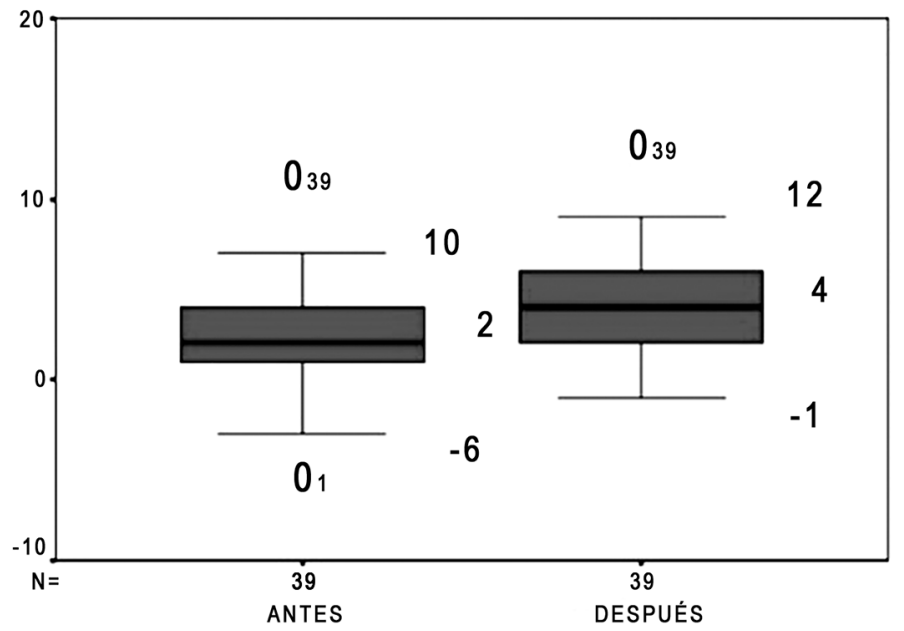

Figura 1

Por otra parte, podemos observar que al evaluar cada uno de los ítems a través de la prueba Wilcoxon, en algunos encontramos un cambio positivo y estadísticamente significativo (Tabla 2).

\begin{tabular}{|c|c|c|c|c|c|c|c|}
\hline \multirow[b]{2}{*}{ Item } & \multicolumn{3}{|c|}{ Pre } & \multicolumn{3}{|c|}{ Post } & \multirow[b]{2}{*}{$p^{*}$} \\
\hline & Correcto & Incorrecto & No sabe & Correcto & Incorrecto & No sabe & \\
\hline $\begin{array}{l}\text { Conoce direccio- } \\
\text { nes electrónicas }\end{array}$ & $\begin{array}{c}26 \\
(67 \%)\end{array}$ & $\begin{array}{c}7 \\
(18 \%)\end{array}$ & $\begin{array}{c}6 \\
(15 \%)\end{array}$ & $\begin{array}{c}32 \\
(82 \%)\end{array}$ & $\begin{array}{c}6 \\
(15 \%)\end{array}$ & $\begin{array}{c}1 \\
(3 \%)\end{array}$ & 0.179 \\
\hline $\begin{array}{l}\text { Identifica la ne- } \\
\text { cesidad de incluir } \\
\text { una contraseña de } \\
\text { acceso }\end{array}$ & $\begin{array}{c}31 \\
(80 \%)\end{array}$ & $\begin{array}{c}2 \\
(5 \%)\end{array}$ & $\begin{array}{c}6 \\
(15 \%)\end{array}$ & $\begin{array}{c}39 \\
(100 \%)\end{array}$ & 0.0 & 0.0 & 0.231 \\
\hline $\begin{array}{l}\text { Define el tipo de } \\
\text { artículo e idioma }\end{array}$ & $\begin{array}{c}14 \\
(36 \%)\end{array}$ & $\begin{array}{c}18 \\
(46 \%)\end{array}$ & $\begin{array}{c}7 \\
(18 \%)\end{array}$ & $\begin{array}{c}9 \\
(23 \%)\end{array}$ & $\begin{array}{c}26 \\
(67 \%)\end{array}$ & $\begin{array}{c}4 \\
(10 \%)\end{array}$ & 0.154 \\
\hline $\begin{array}{l}\text { Acceso a tablas } \\
\text { de contenido }\end{array}$ & $\begin{array}{c}30 \\
(77 \%)\end{array}$ & $\begin{array}{c}1 \\
(3 \%)\end{array}$ & $\begin{array}{c}8 \\
(21 \%)\end{array}$ & $\begin{array}{c}34 \\
(87 \%)\end{array}$ & $\begin{array}{c}5 \\
(13 \%)\end{array}$ & 0.0 & 0.205 \\
\hline $\begin{array}{l}\text { Coloca tema de } \\
\text { busqueda del área }\end{array}$ & $\begin{array}{c}12 \\
(31 \%)\end{array}$ & $\begin{array}{c}14 \\
(36 \%)\end{array}$ & $\begin{array}{c}13 \\
(33 \%)\end{array}$ & $\begin{array}{c}18 \\
(46 \%)\end{array}$ & $\begin{array}{c}19 \\
(49 \%)\end{array}$ & $\begin{array}{c}2 \\
(5 \%)\end{array}$ & 0.231 \\
\hline $\begin{array}{l}\text { Restringe la } \\
\text { búsqueda a } \\
\text { texto completo en } \\
\text { portal }\end{array}$ & $\begin{array}{c}25 \\
(64 \%)\end{array}$ & $\begin{array}{c}6 \\
(15 \%)\end{array}$ & $\begin{array}{c}8 \\
(21 \%)\end{array}$ & $\begin{array}{c}37 \\
(95 \%)\end{array}$ & 0.0 & $2(5 \%)$ & 0.026 \\
\hline $\begin{array}{l}\text { Utiliza términos } \\
\text { MeSH }\end{array}$ & $\begin{array}{c}23 \\
(59 \%)\end{array}$ & $\begin{array}{c}8 \\
(21 \%)\end{array}$ & $\begin{array}{c}8 \\
(21 \%)\end{array}$ & $\begin{array}{c}23 \\
(59 \%)\end{array}$ & $\begin{array}{c}12 \\
(31 \%)\end{array}$ & $\begin{array}{c}4 \\
(10 \%)\end{array}$ & 0.282 \\
\hline
\end{tabular}




\begin{tabular}{|l|c|c|c|c|c|c|c|}
\hline $\begin{array}{l}\text { Utiliza operadores } \\
\text { booleanos }\end{array}$ & $\begin{array}{c}4 \\
(10 \%)\end{array}$ & $\begin{array}{c}25 \\
(64 \%)\end{array}$ & $\begin{array}{c}10 \\
(26 \%)\end{array}$ & $\begin{array}{c}5 \\
(13 \%)\end{array}$ & $\begin{array}{c}30 \\
(77 \%)\end{array}$ & $\begin{array}{c}4 \\
(10 \%)\end{array}$ & 0.641 \\
\hline $\begin{array}{l}\text { Utiliza la herra- } \\
\text { mienta "notes" en } \\
\text { OverDrive }\end{array}$ & $\begin{array}{c}9 \\
(23 \%)\end{array}$ & $\begin{array}{c}8 \\
(21 \%)\end{array}$ & $\begin{array}{c}3 \\
(8 \%)\end{array}$ & $\begin{array}{c}33 \\
(85 \%)\end{array}$ & $\begin{array}{c}3 \\
(8 \%)\end{array}$ & $\begin{array}{c}3 \\
(8 \%)\end{array}$ & 0.001 \\
\hline $\begin{array}{l}\text { Solicita el } \\
\text { prestamo virtual } \\
\text { de libros }\end{array}$ & $\begin{array}{c}7 \\
(18 \%)\end{array}$ & $\begin{array}{c}11 \\
(28 \%)\end{array}$ & $\begin{array}{c}21 \\
(54 \%)\end{array}$ & $\begin{array}{c}17 \\
(44 \%)\end{array}$ & $\begin{array}{c}19 \\
(49 \%)\end{array}$ & $\begin{array}{c}3 \\
(8 \%)\end{array}$ & 0.359 \\
\hline $\begin{array}{l}\text { Utiliza 2 o más } \\
\text { portales en forma } \\
\text { simultánea }\end{array}$ & $\begin{array}{c}6 \\
(15 \%)\end{array}$ & $\begin{array}{c}19 \\
(49 \%)\end{array}$ & $\begin{array}{c}14 \\
(36 \%)\end{array}$ & $\begin{array}{c}21 \\
(54 \%)\end{array}$ & $\begin{array}{c}15 \\
(39 \%)\end{array}$ & $\begin{array}{c}3 \\
(8 \%)\end{array}$ & 0.001 \\
\hline $\begin{array}{l}\text { Solicita apoyo } \\
\text { bibliotecario de } \\
\text { artículos }\end{array}$ & $\begin{array}{c}26 \\
(67 \%)\end{array}$ & $\begin{array}{c}3 \\
(8 \%)\end{array}$ & $\begin{array}{c}1 \\
0(26 \%)\end{array}$ & $\begin{array}{c}38 \\
(97 \%)\end{array}$ & $\begin{array}{c}1 \\
(3 \%)\end{array}$ & 0.0 & 0.231 \\
\hline *Valor de pcalculado con la prueba de X2 de Wilcoxon & \\
\hline
\end{tabular}

Tabla 2. Descripción de resultados por item

A través del análisis de estos resultados pudimos observar que en la mayoría de los ítems hay un ascenso en la puntuación de la prueba posterior contra la anterior, aun cuando algunos usuarios ignoran la respuesta correcta.

En otro aspecto, pudimos demostrar que, posterior a la ALFIN, el usuario elevó su aptitud un $15 \%$ al recordar la dirección electrónica o URL donde se encuentran los RDE del IMSS; asimismo, ascendió hasta un $100 \%$ su habilidad para recordar que el acceso a la plataforma se realiza utilizando contraseñas como su matrícula y primer apellido. Esto representó un cambio positivo ya que ascendió la aptitud un $20 \%$. En cuanto a la aptitud que otorga la estrategia educativa para consultar las tablas de contenido de las revistas y obtener los artículos que de allí le parecen necesarios, se encontró un aumento de $9 \%$.

Por otra parte, el usuario eleva su aptitud para restringir las búsquedas avanzadas ya que hay un aumento de más del $30 \%$ con respecto de la prueba anterior a la ALFIN.

En un aspecto más de su comportamiento informativo, en la utilización de los términos MeSH para restringir la búsqueda se observó un incremento del $1 \%$ en cuanto a utilizar operadores booleanos (and, not, or) para dicha restricción.

Otro de los ítems donde se puede apreciar un importante ascenso en la aptitud posterior a la ALFIN es en la utilización de la BD OverDrive ya que el usuario utilizó mejor las herramientas de esta BD en un $24 \%$ al realizar notas y en un $25 \%$ al solicitar el préstamo virtual de los libros electrónicos.

En el último caso, se refleja una notoria elevación de la aptitud para la utilización simultánea de las $\mathrm{BD}$, pues posterior a la estrategia el usuario 
identificó en un 39 \% que no puede realizar búsquedas simultáneas en las $\mathrm{BD}$, sino en una por ocasión.

Para concluir estos resultados, se observó en el cuestionamiento del caso final que sí existe un aumento en la prueba posterior a la ALFIN, ya que el usuario reconoce en un $30 \%$ más que puede solicitar apoyo bibliotecario para recuperar los artículos de las revistas que no obtuvo en el texto completo inmediato.

Al igual que los resultados positivos de esta investigación, es destacable mencionar que el único ítem que no presentó mejoría con la prueba posterior a la ALFIN es cómo restringir el tipo de artículo y el idioma del mismo, ya que el resultado de la prueba final presentó una disminución de hasta un $13 \%$.

\section{Discusión}

Los resultados observados demostraron que existe una diferencia significativa en la búsqueda y obtención de la información una vez que los residentes tomaron la ALFIN o estrategia educativa por parte del personal bibliotecario, puesto que aumentó su aptitud para realizar búsquedas de información además de observarse que, posterior a la estrategia, adquirieron habilidades que les permitieron obtener el texto completo de los artículos de revistas y capítulos de libros electrónicos dentro del portal de la Coordinación de Educación en Salud del IMSS.

El aumento de la aptitud del usuario es de gran conveniencia para el IMSS ya que al conocer y utilizar las BD que se ofrecen a través de la CDES se favorece su utilización y aprovechamiento en beneficio de su formación académica y actualización continua de la profesión.

La formación de usuarios ante el recurso documental electrónico que ofrecen las BD especializadas en su área presenta un importante hallazgo, como se demuestra al vincular estos resultados con los que señala un estudio realizado a profesores de la Escuela de Ciencias de la Salud en la Universidad de los Andes, en Venezuela. En este estudio se encontró que la mayoría de los encuestados desconocen en un $71 \%$ los mecanismos de acceso a las BD especializadas (Espinoza, Rincón y Chacín, 2006: 32). También podemos observar que este resultado no dista mucho del resultado inicial o anterior al otorgamiento de la ALFIN de la investigación presente, ya que los encuestados demostraron ignorar estos mismos mecanismos en un $80 \%$ al obtener un resultado de 93 puntos (20\%) de los 468 que representaban el total de una codificación general. 
Como señala la ALA, no basta con disponer de información en cualquiera de sus medios o formatos, más aún con el cúmulo de información con que contamos a través de Internet, debido básicamente a la falta de evaluación en la "autenticidad, validez y confiabilidad" (ALA, 2010). Si aunado a esto reconocemos la dificultad que representa para el usuario encontrar eficazmente lo que cubre su necesidad de información, los retos se duplican. Esto nos lleva a requerir de aptitudes que permitan el uso de esa información aplicada a todas las disciplinas del conocimiento y en todos los niveles de educación. Esto también lo podemos observar en los resultados de Loyola Contreras (2006: 7), quien estudió el comportamiento informacional para la búsqueda y recuperación de información a través de Internet a estudiantes de Pedagogía de la Universidad de Playa Ancha de Ciencias de la Educación en Chile, donde identificó que sus usuarios consideran necesaria la ALFIN en un $67 \%$ y muy necesaria en un $32 \%$.

El otorgamiento de una ALFIN adecuada en las BD apropiadas para realizar la búsqueda y recuperación de información le permite al educando no sólo dominar esas estrategias, sino ir formando sus propios métodos de búsqueda a fin de que le otorguen la información que necesita y requiere. Por otra parte, se consideró lo señalado por Thompson y Blankinship (2015: 31) al otorgar la ALFIN al inicio de la formación académica -en el primer grado de la residencia y en los primeros meses de su ingreso- pues, como describen las autoras, hay más impacto en las habilidades que adquieren los usuarios después de una ALFIN.

Aunque los resultados se han mostrado favorables para esta investigación, también es acertado reconocer que no todos los aspectos tratados en el instrumento de medida otorgaron un valor de importancia significativa. Esto permite identificar que la confiabilidad y validez del mismo debió realizarse de manera más eficiente. Estos aspectos que no otorgaron una medida útil se han convertido en áreas de oportunidad para mejorar la estrategia educativa que se brinda reafirmando la retroalimentación de los servicios del Centro de Documentación. Al mejorar el instrumento en cuestión se podrá difundir para que las UI del IMSS lo utilicen como una herramienta que permita evaluar los cursos que están programados en cada unidad, especialmente porque esta institución cuenta con la red más grande de UI o bibliotecas médicas de México y por tanto con la mayor cantidad de usuarios de las áreas de la salud.

Como último aspecto a discutir es necesario destacar que esta investigación se realizó en 2010, esperando tomar una nueva muestra sólo al presentarse cambios en las BD o la plataforma digital de EDUMED, a través de la que se suscriben las colecciones digitales o RDE. Estos cambios se iniciaron 
en 2015, pero se concretaron hasta 2016 con la inclusión del IMSS al Consorcio Nacional de Recursos de Información Científica y Tecnológica (CONRICYT). Esto permitió modificar la ALFIN con una nueva estrategia de búsqueda y recuperación que posibilitará al usuario buscar y obtener mayor cantidad de información y realizar una diseminación que le otorgue resultados más oportunos y pertinentes a sus necesidades de información actuales. Asimismo, permitirá medir nuevamente la aptitud del usuario del recurso documental electrónico y mantener una innovación en la capacitación, formación o educación de usuarios de las UI del SIBIMSS.

\section{CONCLUSIONES Y RECOMENDACIONES}

Con la implementación de la ALFIN o estrategia educativa titulada Aprovechamiento de las fuentes electrónicas a través del Portal de Educación del IMSS:

1. Los usuarios presentaron una mayor aptitud para la realización de búqueda y recuperación del RDE a través del portal de EDMED.

2. El otorgamiento de este tipo de ALFIN en los programas de formación de usuarios que se realizan en el CRDS del IMSS permitió que los médicos residentes no sólo conozcan la existencia de este recurso documental electrónico al que tienen acceso, sino que además conozcan las ventajas y el modo de aprovecharlo para las investigaciones documentales que realizan como práctica de su formación académica y posterior práctica diaria profesional.

3. El describir el comportamiento informacional de los usuarios estudiados, otorgó una medida para establecer evaluaciones constantes a las estrategias de búsqueda y recuperación de información en las áreas de la salud.

\section{Agradecimientos}

Al Centro de Documentación en Salud en el Centro Médico Nacional del Noroeste del Instituto Mexicano del Seguro Social en ciudad

Obregón, Sonora. México; por las facilidades otorgadas para la realización de este trabajo de investigación. 


\section{REFERENCIAS}

ALA (American Library Association), Association of College and Research Libraries. 2010. Normas sobre aptitudes en el acceso y uso de la información de la ensenanza superior. Fecha de consulta: mayo de 2010. http://www.ala.org/ala/mgrps/ divs/acrl/standards/informationliteracycompetencystandards.cfm

De Bibliotecarios, A. A. 2000. "Normas sobre aptitudes para el acceso y uso de la información en la educación superior". Boletín de la AAB 15 (60). Fecha de consulta: mayo de 2010. http://www.ala.org/ala/mgrps/divs/acrl/standards/informationliteracycompetencystandards.cfm

Castro Montoya, M. R. 2008. "El servicio de consulta y sus recursos documentales desde una perspectiva de los usuarios del Centro Regional de Documentación en Salud del Hospital de Especialidades No.1 de Ciudad Obregón, Sonora, IMSS”. Tesis, ENBA, Cd. Obregón, Sonora, México.

De la Cruz Solis, M., T. González Méndez y T. Vivás Jiménez. 2007. “El papel de una biblioteca de área en la formación de los profesionales”, en Proceedings XII Jornadas Nacionales de Información y Documentación en Ciencias de la Salud, 1-10. Fecha de consulta: diciembre de 2008. http://eprints.rclis.org/10825/

Espinoza, K., A. G. Rincón y B. Chacín. 2006. "Búsqueda de información en la Web por profesionales de salud en una universidad venezolana: un estudio transversal". El Profesional de la Información 15 (1): 28-33.

González Teruel, A. 2011. "Investigación sobre usuarios y realidad de la gestión de unidades de información: interacciones posibles y necesarias”, en $2^{\circ}$ Seminario de Estudios da Informação, Niteroi, R. J., Brasil. Fecha de consulta: agosto de 2016. http://eprints.rclis.org/18526

IMSS (Instituto Mexicano del Seguro Social). Centro Regional de Documentación en Salud. 2009. Programa para la formación de usuarios de la información 2009. México: IMSS.

Lancaster, F. W. 2003. "Evaluación de la formación bibliográfica de usuarios”, en Evaluación de la biblioteca, 239-274. Madrid, España: ANABAD.

Licea, J. 2007. "La evaluación de la alfabetización informacional: principios, metodologías y métodos”. Anales de Documentación 10: 215-232.

López Espinosa, J. A. y S. Díaz del Campo. 1996. "La educación y formación de los médicos de la familia como usuarios de la información". ACIMED 4(2). Fecha de consulta: diciembre de 2008. http://eprints.rclis.org/2286/1/aci03296.htm

Loyola Contreras, E. 2006. "Uso inteligente de Internet: experiencia en la Universidad de Playa Ancha de Ciencias de la Educación”. Biblios. 7(3). Fecha de consulta: noviembre de 2007. http://dialnet.unirioja.es/servlet/fichero_articulo?codigo $=1431578$ \&orden $=0$

Llanusa-Ruiz, S. B., C. N. Rojo-Pérez, Y. Sarduy-Domínguez, P. A. Urra-González. 2015. "Herramientas para el análisis cualitativo en estudio del empleo de la información científica por usuarios de Infomed”. Educación Médica Superior 29 (2): 324-339.

Perello, G. J. 2005. "El usuario de bibliotecas ante los nuevos soportes y los nuevos servicios: el usuario electrónico". Revista General de Información y Documentación 15 (2): 39-50. Fecha de consulta: diciembre de 2008. http://revistas.ucm.es/ byd/11321873/articulos/RGID0505220039A.PDF 
Thompson, L. y L. A. Blankinship. 2015. "Teaching information literacy skills to sophomore-level biology majors". Journal of Microbiology E Biology Education 16 (1): 29-33.

Universidad Complutense de Madrid y Pérez Iglesias, J. 2007. La alfabetización informacional en las universidades: una propuesta de actuación para la Biblioteca de la Universidad Complutense de Madrid. Documentos de Trabajo en la Escala de Facultativos de Archivos y Bibliotecas de la UCM 4. Fecha de consulta: diciembre de 2008. http://www.ucm.es/BUCM/biblioteca/doc10023.pdf

Viniegra Velázquez, L. 2002. "Una educación para la participación”, en Educación y crítica, el proceso de elaboración del conocimiento, 108-142. México: Paidós.

\section{Anexo}

\section{Encuesta}

La presente encuesta se realiza para evaluar parte de los servicios que se le otorgan a través del Centro de Documentación, por lo que amablemente se le solicita su apoyo para contestar las siguientes afirmaciones y ante lo cual agradecemos de antemano su colaboración.

Usted pertenece al Grado de la Residencia Médica de:

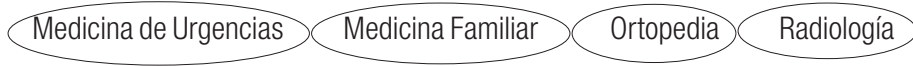

Favor de responder en cada línea frente a las afirmaciones:

F= Falso: cuando no esté de acuerdo con la afirmación.

V= Verdadero: cuando esté de acuerdo con la afirmación.

$\mathrm{N}=$ No sé: cuando ignore ssi la afirmación es falsa o verdadera.

- Partiendo del URL o dirección electrónica de la Coordinación de Educación del IMSS, se puede tener acceso a los documentos adquiridos para la utilización de su personal; donde una vez que se ingresa a esta dirección electrónica, se abre una ventana en la que hay que oprimir el botón "Servicios Electrónicos" y al abrirse la siguiente ventana hay que dar el clic en "Publicaciones Electrónicas" llegando a la ventana que solicita colocar las claves de acceso.

- Es ante esta afirmación una situación favorable: que una vez que se está en el navegador, se escriba la dirección electrónica: http://edumed.imss.gob.mx para poder ingresar a la página de la Coordinación(Indicador A) agregar la matrícula y el apellido para poder ingresar a los portales de libros y revistas (Indicador B) 
- Al realizar una búsqueda documental ya que se ha ingresado a la ventana que ofrece los distintos botones o ligas de los portales adquiridos, se coloca el tema de búsqueda en el área que indica "Digite el texto" y se oprime el botón "Buscar".

- Es apropiado según el párrafo anterior que se obtengan: artículos de revisión y en idioma español (Indicador C) el acceso a los libros y revistas de los distintos portales, para poder navegar en sus tablas de contenido y los artículos que contienen (Indicadores B y C)

- Buscando libros y revistas que contengan algún tema de interés, dentro de los portales electrónicos adquiridos por el IMSS, se vuelve necesario utilizar las distintas herramientas que contienen estos portales para poder obtener artículos o capítulos que verdaderamente aporten información útil a la necesidad de información. Es por ello que se pueden obtener resultados más acertados cuando se realiza la búsqueda de un tema en el área de "Búsquedas Avanzadas" aprovechando los limitantes y condicionantes como buscar en el área de "Título", elegir "Documentos Disponibles a Texto Completo" y solicitar un rango de tiempo que abarque sólo resultados entre los 5 últimos años.

- Ante lo expuesto es adecuado que se localicen:

artículos y capítulos que pueden o no contener las palabras que forman el tema de búsqueda en el título. (Indicadores $C$ y D)

sólo aquellos artículos o libros que están disponibles a texto completo, adquiridos por el IMSS(Indicadores C y E)

- Al realizar la búsqueda de documentos sobre Medicina, Salud o afines, contamos con una útil herramienta como es un vocabulario controlado llamado MeSH (por sus siglas inglesas traducidas como Encabezamientos Médicos de Materia) los cuales permiten obtener mejores resultados al buscar documentación de un tema en particular. De igual manera la búsqueda se vuelve más productiva cuando se utilizan los operadores booleanos 0 palabras de unión, disyunción o truncamientos como "and, not, or" o sus traducciones al español "y, no, o" sobre todo con la utilización de términos compuestos como ocurre frecuentemente en medicina y salud, o cuando se requiere que de determinado tema se encuentre sólo lo referente a diagnóstico, tratamiento, estadísticas, etc.

- Es una acción apropiada en este caso: buscar "Factores de riesgo para infarto agudo del miocardio" colocando en el área de "Título" Infarto Agudo del Miocardio y Factores de Riesgo (Indicadores D y F) se busca "Diabetes Gestacional" a través de la búsqueda Diabetes y Embarazo (Indicadores D y F)

- En el portal "OverDrive" se puede obtener el "Préstamo Virtual" de algunos libros que se encuentran disponibles a través de ingresar al portal y realizar la búsqueda; posteriormente cuando se tienen los resultados y se requiere un préstamo, se debe dar clic o ingresar sobre el título o la portada del libro. Enseguida aparecerán las características de la obra y del préstamo, aclarando si existen obras disponibles para préstamo en "Copias en Biblioteca: 
1". Enseguida se oprime el botón "Agregar mis e-Books" y después "Proceder con el Préstamo" con lo cual se da por aceptado el préstamo virtual por el tiempo señalado (que puede variar de 1 a 3 días). Se sigue con la aceptación de "Confirmar el Préstamo" y posteriormente se tiene acceso al contenido del libro con "Descargar"

- Es correcto que cuando se descargue el libro:

se tenga acceso a su contenido y se pueden hacer "notas", "resaltado o subrayado de texto" dentro del libro en préstamo (Indicador G)

solicitar en préstamo virtual todos los libros que se deseen para su utilización simultánea (Indicador G)

- La utilización de los distintos portales permiten la opción de utilizar sus libros y revistas, realizando las búsquedas que partan de un tema de interés en el área de la salud; sin embargo, es importante tener presente que si se han buscado en todos los portales documentos sólo con "Texto Completo" y no hubo resultados, es también una gran ventaja poder hacer una búsqueda bibliográfica de artículos sin texto completo, pero que me ofrezca los resúmenes que posiblemente sirvan al tema de interés, y tratar de localizarlos por otros medios y servicios del propio CRDS y gratis.

- Es apropiado que cuando se realiza la búsqueda documental: se pueda realizar simultáneamente en dos o más portales el mismo tema u otro distinto (Indicador B) solicitar al personal bibliotecario los artículos que no están a texto completo, pero que se requieren para su utilización; otorgando para ello la lista de referencias bibliográficas (Indicador E)

\section{¡De nuevo GRACIAS por su colaboración!}

Para citar este texto:

Castro Montoya, Maria del Rubí, Mariano-Padilla Mendoza y José Manuel Ornelas-Aguirre. 2018. "Aptitud de médicos residentes para la búsqueda y recuperación de información”. Investigación Bibliotecológica: archivonomia, bibliotecología e información 32 (75): 145-161.

http://dx.doi.org/10.22201/iibi.24488321xe.2018.75.57965 